\title{
Aprendizaje de lenguas modernas basado en redes sociales
}

\author{
Germán RUIPÉREZ GARCÍA \\ Universidad Camilo José Cela \\ german@ruiperez.org \\ José Carlos García CABRERO \\ Universidad Nacional de Educación a Distancia \\ contacto@josecarlosgarciacabrero.es
}

Recibido: 30/07/2012

Aceptado: 23/10/2012

\begin{abstract}
Resumen
En los modelos de formación on-line cada vez se está extendiendo más el uso de redes sociales, de tal manera que muchos autores utilizan el término Social Learning para definir esta nueva modalidad de aprendizaje virtual. Con el fin de evaluar el potencial de las redes sociales en el aprendizaje de lenguas modernas, y, por otra parte, compararlo con el de las plataformas de elearning convencionales, se llevó a cabo una investigación en la asignatura "Metodología práctica para la enseñanza en línea de lenguas extranjeras", en la que se utilizó la red social Google+ en el grupo experimental, y la plataforma de la UNED (.LRN) en el grupo de control. Los resultados finales confirmaron una opinión muy favorable de los usuarios en cuanto a usabilidad y potencial de las redes sociales en el aprendizaje on-line. No obstante, no se pudo demostrar una eventual ventaja de las redes sociales frente a las LMS convencionales, a pesar de que el trabajo colaborativo que se pudo realizar con la red social Google+ hubiera sido prácticamente imposible de llevar a cabo en la plataforma institucional.
\end{abstract}

Palabras clave: aprendizaje on-line, lenguas modernas, redes sociales, Google+

\section{Learning modern languages using social networks}

\begin{abstract}
The use of social networks is spreading more and more, so that many authors use the term Social Learning for this new form of learning. In order to evaluate the potential of social media in learning modern languages, and, moreover, to compare it with that of conventional eLearning platforms, we carried out a research on the subject "Methodology for teaching practice online foreign language ", in which we used the social network Google+ in the experimental group and the UNED platform (.LRN) in the control group.

The final results confirmed a very favourable opinion of the users in terms of usability and about potential of social media in online learning. However, we were unable to demonstrate any advantages of social networks compared to conventional LMS, although collaborative work that could be performed with the social network Google+ would have been practically impossible to carry out in the institutional platform.
\end{abstract}

Keywords: online learning, modern languages, social networks, Google+

\section{Referencia normalizada}

RUIPÉREZ GARCÍA, Germán y GARCÍA CABRERO, José Carlos (2012): “Aprendizaje de lenguas modernas basado en redes sociales". Estudios sobre el mensaje periodístico. Vol. 18, núm. especial noviembre, págs.: 821-827. Madrid, Servicio de Publicaciones de la Universidad Complutense.

Sumario: 1. Introducción. 2. Diseño de la investigación. 3. Desarrollo del proyecto; 3.1. Encuesta inicial; 3.2. Configuración básica de Google+ y del entorno personal de aprendizaje; 3.3. Formación de los grupos y desarrollo del trabajo; 3.4. Publicación y debate sobre los documentos generados por cada grupo; 3.5. Encuesta final. 4. Conclusiones. 5. Referencias bibliográficas. 


\section{Introducción}

Las redes sociales en Internet han experimentado un crecimiento espectacular en los últimos años. Por citar un ejemplo representativo en el que los datos son elocuentes, en el primer trimestre de 2012, y con motivo de su salida a bolsa, la documentación oficial presentada a la Securities and Exchange Commission hablaba de 901 millones de usuarios activos mensuales de Facebook Inc. (2012, web), un 33\% más que en el mismo periodo de 2011.

Por ello, y como la educación no puede ser ajena a estas tendencias, cada vez son más los defensores de la integración en la práctica docente en línea de las redes sociales (Dalsgaard, 2006: web; Siemens, 2007: web; o Panckhurst y Marsh, 2011: 233252), en sustitución de las clásicas plataformas virtuales o Learning Management Systems (LMS), como Moodle o Blackboard, por citar dos de las más conocidas.

Como no tenemos constancia de la existencia de estudios empíricos relevantes que avalen esta tendencia, al menos en el ámbito español, el equipo investigador decidió hacer un estudio de campo con los alumnos de la asignatura "Metodología práctica para la enseñanza en línea de lenguas extranjeras" ( $2^{\circ}$ cuatrimestre) 2011/2012 del Máster Universitario en "Tecnologías de la Información y Comunicación en la Enseñanza y Tratamiento de las Lenguas" de la UNED, incorporando a su docencia la red social Google+, y manteniendo en paralelo un grupo de control en la plataforma oficial de la UNED, denominada aLF (adaptación de .LRN).

Los principios básicos que, según los diversos investigadores citados anteriormente, sustentan este enfoque son dos:

1. El elevado número de usuarios, y partir del principio natural de que el estudiante trabaje con herramientas tecnológicas que ya domina. Por dar algunos datos recientes, según Vizoso Martín (2012: 181-203), más del 80\% de los adolescentes españoles conoce y utiliza las redes sociales, y más del $50 \%$ las consulta varias veces al día, siendo frecuente que se mantengan perfiles activos en dos o más redes sociales, particularmente Facebook y Tuenti.

2. Mediante el uso de las redes sociales el estudiante puede configurar su propio entorno personal de aprendizaje (Personal Learning Environment, PLE), lo que le proporciona una flexibilidad de la que, en general, no dispone en un entorno académico más controlado y formal, característico de las plataformas virtuales (LMS).

No debemos olvidar que, por otra parte, las redes sociales son instrumentos diseñados específicamente para facilitar todo tipo de interacciones, por lo que esta capacidad las convierte en herramientas especialmente apropiadas para la resolución cooperativa de tareas, uno de los paradigmas más apropiados para la adquisición de competencias en el EEES, pues se trabajan no solo las relacionadas con la materia en cuestión, sino también las sistémicas y transversales, según los estudios de Miguel Díaz (2006: 102-105).

En resumen, nuestra hipótesis de trabajo para la investigación se formularía de la siguiente manera: la realización de actividades cooperativas mediante las redes sociales constituye un enfoque adecuado para el aprendizaje en línea en educación superior. 


\section{Diseño de la investigación}

A partir de nuestra hipótesis de trabajo, se identificaron dos objetivos principales:

1. Probar la utilidad y potencial de las redes sociales como herramientas de docencia en línea en la enseñanza universitaria.

2. Comparar las interacciones y percepciones de los estudiantes en el uso de las mismas, frente a las producidas en el entorno académico formal basado en una plataforma virtual (LMS).

Nuestra propuesta partía de principios pedagógicos constructivistas, en los que la base es "aprender haciendo" (learning by doing), de forma que el papel activo y la responsabilidad del aprendizaje recaen sobre el estudiante.

Consideramos siempre un enfoque de investigación-acción en el sentido expresado por Elliot (2000: 5) de que "se relaciona con los problemas prácticos cotidianos experimentados por los profesores con el objetivo de profundizar su comprensión mediante una postura exploratoria".

Para lograr una mayor motivación y participación de los estudiantes, el estudio de campo se vinculó a un trabajo cooperativo, en el que, a partir de los intereses personales y perfiles profesionales de los estudiantes que decidieron voluntariamente participar, se articularon cinco grupos con el objetivo de redactar partes comunes al trabajo final obligatorio de evaluación de la asignatura ${ }^{1}$.

No se debe olvidar que, dado que la materia de estudio era la metodología del aprendizaje en línea de lenguas extranjeras, la propia experiencia llevada a cabo en la investigación proporcionaba un feedback implícito inmediato a todos los estudiantes, y les hacía reflexionar sobre sus propias prácticas docentes y planteamientos iniciales, enriqueciendo su aprendizaje. De esta manera eran ellos los principales protagonistas de una experiencia similar a la que se les proponía desarrollar con sus alumnos.

\section{Desarrollo del proyecto}

La investigación se llevó a cabo en las siguientes fases:

1. Encuesta inicial: perfil previo y temas de interés.

2. Configuración básica de Google + y del entorno personal de aprendizaje.

3. Formación de los grupos y desarrollo del trabajo.

4. Publicación y debate sobre los documentos generados por cada grupo.

5. Encuesta final.

En primer lugar, y utilizando las herramientas de comunicación del curso virtual estándar proporcionado por la UNED, se informó a los estudiantes de la posibilidad de participar en el proyecto y de las características principales del mismo.

${ }^{1}$ Dicho trabajo final consistía en el diseño e implementación de una actividad on-line para trabajar una competencia determinada en la lengua de trabajo que el estudiante enseña a sus alumnos: se trata de una asignatura de Máster Universitario en la que prácticamente la totalidad de los estudiantes son, a su vez, docentes de una lengua extranjera en diferentes niveles educativos. 
Es necesario mencionar que, además del experimento aquí reseñado, se siguió paralelamente la dinámica habitual en el curso virtual estándar en el LMS propio de la universidad, denominado aLF.

Por ello, el primer paso que tuvieron que dar los estudiantes que voluntariamente quisieron participar fue inscribirse rellenando una encuesta inicial, en la que, además de recoger los datos personales básicos, teníamos dos propósitos:

1. Disponer de un perfil previo de los estudiantes.

2. Identificar áreas de interés comunes y lenguas de trabajo para poder formar grupos homogéneos.

En las encuestas, además de las preguntas dirigidas a valorar el grado de cumplimiento de los objetivos de investigación previstos, se incluyeron ítems para recoger impresiones pre y post sobre el trabajo en grupo.

Respecto a la elección de Google+ frente a otras redes sociales quizás más populares, el factor determinante fue el ecosistema de herramientas asociadas, que permitía tener en un entorno integrado documentos compartidos a modo de wiki, sesiones síncronas de chat o de audio, videoconferencia, etc.

También resultó determinante la manera de organizar los contactos y mantener la privacidad mediante círculos de Google+, que permitía saber en todo momento qué personas estaban viendo una información y que, además, permitía relaciones asimétricas seguidor-seguido como en Twitter, y al contrario que otras redes sociales como Facebook o Tuenti.

\subsection{Encuesta inicial}

De los 31 alumnos matriculados en la asignatura, 19 decidieron participar en el proyecto. Pensamos que esta notable acogida tuvo que ver con la ya referida popularidad de las redes sociales, pues, de hecho, el 100\% de los diecinueve participantes declararon utilizar con cierta asiduidad al menos una red social.

El perfil tecnológico de los participantes era medio alto ${ }^{2}$ y, en consecuencia, pensamos que, con un breve tutorial y un mínimo periodo de adaptación a la herramienta, sería suficiente formación para que no hubiera problemas técnicos.

La principal dificultad fue la gran variedad de lenguas de trabajo propuestas (inglés, francés, alemán, italiano y español para extranjeros) y los distintos niveles (desde A1 hasta $\mathrm{C} 1$ o fines específicos), por lo que, finalmente, no todos los grupos pudieron formarse con la homogeneidad deseada en este sentido.

En cuanto a los temas de interés general, los más populares resultaron ser el uso didáctico de las propias redes sociales y de herramientas Web 2.0 (blogs, wikis, etc.). En consecuencia, y a la vista de la diversidad de las lenguas de trabajo, se formaron cinco grupos, uno para cada uno de los temas más mencionados por ellos: comprensión oral (YouTube, podcast, etc.), expresión oral (Skype, etc.), redes sociales, Twitter y wikis.

${ }^{2} \mathrm{Al}$ ser una asignatura del segundo cuatrimestre, todos los alumnos han cursado las materias de formación básica en TIC en el primero. 


\subsection{Configuración básica de Google + y del entorno personal de aprendizaje}

Como ya hemos mencionado anteriormente, los datos de la encuesta inicial nos dieron un perfil tecnológico medio alto, por lo que la formación y la configuración básica fueron sencillas y rápidas.

Pedimos a los estudiantes que crearan nuevas cuentas de Gmail, exclusivas para el proyecto $^{3}$, y que configuraran Google+ añadiendo un círculo común para todos los participantes, incluyendo al equipo docente.

En esta fase inicial, y después de haberles facilitado dos tutoriales interactivos, les pedimos también completar el perfil en la red social y experimentar con las distintas herramientas, y, muy particularmente, con la videoconferencia denominada "Quedadas". Hicimos también hincapié en las posibilidades de trabajo en grupo a través de documentos compartidos.

\subsection{Formación de los grupos y desarrollo del trabajo}

Una vez completada la fase inicial de utilización de Google+, se articularon los cinco grupos de trabajo con el siguiente esquema básico inicial:

- Un círculo en $\mathrm{G}+$ para las comunicaciones internas del grupo 4 .

- Un documento en edición compartida a modo de wiki para la realización del trabajo. Este documento, con un esquema fijo y una bibliografía de partida para cada grupo, fue proporcionado por el equipo docente e investigador.

También se recomendó a cada grupo hacer una sesión síncrona inicial a través de la herramienta "Quedadas", para fijar determinados roles en el grupo (coordinador, bibliotecario, etc.) y para poder repartir, de forma rápida y efectiva, las tareas entre los distintos miembros. Por nuestra experiencia previa en cursos on-line, el consenso en esta fase inicial se consigue de manera más efectiva y rápida en sesiones síncronas, antes que a través de correos electrónicos o tablones tipo foro.

El proyecto se desarrolló, en líneas generales, según lo esperado, aunque naturalmente hubo divergencias entre los ritmos o formas de organización de trabajo de cada uno de los grupos.

Durante esta fase, podemos destacar positivamente la propia evolución del grupo y de cada alumno en la configuración del entorno de aprendizaje, mediante otros documentos compartidos según las tareas acordadas, la utilización de herramientas ajenas a la red social en determinados casos, etc.

Destacamos, de igual manera, el gran espíritu de colaboración entre todos los participantes, compartiendo enlaces y documentos, soluciones a problemas comunes, etc. Verdaderamente se dio un aprendizaje social en el sentido más amplio del término.

3 Dado que necesariamente había que hacer públicas las cuentas de correo entre los distintos participantes, se tomó esta decisión para mantener la privacidad de las cuentas habituales dado que son datos confidenciales.

4 Se mantenía además el círculo general para compartir información de interés común para todos los participantes y el equipo docente tenía presencia en todos los círculos. 
Como era previsible, el mayor inconveniente fue la distinta dedicación de los miembros del grupo, pues prácticamente todos tenían obligaciones profesionales, lo cual tuvo como consecuencia una evolución dispar de los grupos, aunque finalmente todos consiguieron alcanzar los objetivos mínimos previstos.

No se dieron, sin embargo, situaciones conflictivas dentro de los grupos, y cada participante asumió los papeles y compromisos inicialmente pactados por ellos.

\subsection{Publicación y debate sobre los documentos generados por cada grupo}

Para finalizar los proyectos, el coordinador de cada grupo hizo público el documento final para compartirlo con el resto de grupos, con el fin de iniciar un debate sobre cada uno de ellos.

Aunque inicialmente la idea fue bien acogida, debemos señalar que el debate producido fue escaso. Pensamos que la proximidad de las evaluaciones finales restaron quizás el tiempo necesario para esta fase. El éxito fue relativo, aparte de que los comentarios al trabajo del resto de grupos fueron poco numerosos y de relativa poca profundidad. Es, sin lugar a dudas, la fase que tiene que ser mejorada en los siguientes cursos.

\subsection{Encuesta final}

De los 19 participantes, solo 11 respondieron la encuesta final. Las valoraciones que hicieron son, en general, positivas: el 100\% de los participantes pensaron que la idea propuesta era buena y adecuada a los objetivos de la asignatura, y el 90,9\% creyó que la experiencia había sido positiva, valorando el plan de trabajo propuesto y los aspectos eminentemente prácticos del proyecto.

Entre los aspectos más negativos, un 36,7\% declaró no haber dedicado todo el tiempo que hubiera querido, y el 27,3\% manifestó que le hubiera gustado que se hubiera producido más comunicación e intercambio de ideas entre todos los miembros de los distintos grupos, aunque sí consideraron adecuadas las interacciones producidas dentro de su grupo.

Por otra parte, también se valoró muy positivamente el trabajo cooperativo a pesar de las dificultades intrínsecas. E1 91,9\% declaró que fue muy enriquecedor, aunque al $63,6 \%$ le resultó difícil integrarlo en el día a día. Además, para el 81,8\% el esfuerzo mereció la pena.

Desde el punto de vista tecnológico, Google+ se valoró positivamente: el $81,8 \%$ manifestó que era una herramienta adecuada, y al 90,9\% le resultó sencilla su utilización. Las herramientas más valoradas fueron los documentos compartidos y las quedadas ( $90 \%$ de opiniones positivas en ambos casos), mientras que la menos valorada fue la organización en círculos de los contactos. A pesar de la excelente valoración, al $72,7 \%$ le hubiese gustado probar otras redes sociales alternativas.

Para finalizar, y aunque al $81,9 \%$ le hubiera gustado que se hubiera utilizado Google + en otras asignaturas o que se hubieran probado otras redes sociales, solo el $54,6 \%$ declaró haberse sentido más cómodo utilizando Google+ que la plataforma aLF de la UNED, y solo el $36,4 \%$ creyó que la red social era más potente que el LMS. Es notorio también que el 18,2\% seleccionó la opción "No sabe/No contesta" en estas 
preguntas, una opción prácticamente no utilizada como respuesta en el resto de preguntas de la encuesta final.

\section{Conclusiones}

A pesar de que la asignatura y el experimento acaban de terminar, y está pendiente todavía un análisis exhaustivo, las primeras conclusiones provisionales parecen confirmar una opinión generalizada muy favorable al uso de Google+ en cuanto a usabilidad y potencial, corroborando de esta forma nuestra hipótesis de trabajo inicial sobre la adecuación de las redes sociales a la docencia en línea en educación superior, articulada mediante la realización de trabajos cooperativos.

Sin embargo, no se constata una opinión clara sobre la eventual superioridad o ventajas de esta red social, Google+, frente a la plataforma oficial de la universidad, a pesar de que un trabajo cooperativo de estas características hubiera sido prácticamente imposible de llevar a cabo en dicha plataforma virtual, dadas las limitaciones intrínsecas de las herramientas disponibles en la misma.

\section{Referencias bibliográficas}

DALSGAARD, Christian (2006): "Social software: E-learning beyond learning management systems". European Journal of Open, Distance and E-Learning, Vol. II, 2006. Disponible en http://www.eurodl.org/materials/contrib/2006/Christian_Dalsgaard.htm. [Fecha de consulta: 10 de septiembre de 2012].

ELLIOT, John (2000): La investigación-acción en educación. Madrid, Morata.

FACEBOOK INC (2012): Amendment No. 4 to Registration Statement. U.S. SEC. Disponible en http://www.sec.gov/Archives/edgar/data/1326801/0001193125121 75673/d287954ds1a.htm. [Fecha de consulta: 8 de septiembre de 2012].

MIGUEL DÍAZ, Mario de (coord., 2006): Metodologías de enseñanza y aprendizaje para el desarrollo de competencias. Orientaciones para el profesorado universitario ante el Espacio Europeo de Educación Superior. Madrid, Alianza Editorial.

PANCKHURST, Rachel y MARSH, Debra (2011): “Utilización de redes sociales para la práctica pedagógica en la enseñanza superior impartida en Francia: perspectivas del educador y del estudiante". Revista de Universidad y Sociedad del Conocimiento, 2011, Vol. 8, $\mathrm{n}^{\circ}$ 1, pp. 233-252. Disponible en http://hdl.handle.net /10609/8931. [Fecha de consulta: 9 de septiembre de 2012].

SIEMENS, George (2007): "Future of learning: LMS o SNS?" Disponible en: http://www.connectivism.ca/?p=192. [Fecha de consulta: 6 de julio de 2012].

VIZOSO MARTÍN, Clara María (2012): Estudio comparativo del uso de las redes sociales por los adolescentes en tres Comunidades españolas: Andalucía, Cataluña y Madrid. Tesis Doctoral sin publicar. Universidad Camilo José Cela, Madrid. 


\section{Germán RUIPÉREZ}

Catedrático de eLearning de la UCJC - España

Email: german@ruiperez.org

Dirección postal: UCJC - Castillo de Alarcón, 49 - 28692 Villanueva de la Cañada (Madrid)

\section{José Carlos GARCÍA-CABRERO}

Profesor de la UNED - España

Email: contacto@josecarlosgarciacabrero.es

Dirección postal: UNED - Facultad de Filología. Senda del Rey, 7. 28040 - Madrid 